\title{
Should internal mammary lymph node sentinel biopsy be performed in breast cancer: a systematic review and meta- analysis
}

\author{
Jing Gong ${ }^{1}$, Yongfu Yu², Gaosong $\mathrm{Wu}^{1}$, Congyao $\mathrm{Lin}^{1^{*}}$ (D) and Xin $\mathrm{Tu}^{3}$
}

\begin{abstract}
Purpose: Results from studies of internal mammary lymph node sentinel biopsy are inconsistent.

Methods: A comprehensive literature search was conducted in MEDLINE, EMBASE, Scopus, Cochrane database, and Clinical Trials. Studies reporting the rate of internal mammary lymph node sentinel biopsy (IMN-SLNB) positivity were identified. We performed pooled proportion meta-analysis using random-effects meta-analyses. The correlation of IMN and axillary lymph node (AXN) metastasis was also investigated.

Results: After application of inclusion and exclusion criteria, a total of 18 articles (total number of patients $=2427$ ) were included. The pooled estimate for IMN-SLNB positivity rate was 15\% (95\% confidence interval (CI) 12-17\%). Significant between-study heterogeneity was observed. Our results indicate that axillary lymph node metastasis is a strong predictor of IMN involvement (OR 6.01, 95\% Cl, 3.49, 10.34).

Conclusion: Internal mammary lymph nodes metastasis might be underestimated. Patients with positive axillary lymph nodes have a higher risk of internal lymph nodes metastasis. As a result, IMN-SLNB might be considered in these patients. Future work needs to be done to assess whether pathological confirmed IMN metastasis can affect patients' survival.
\end{abstract}

Keywords: Internal mammary lymph node, Sentinel lymph node biopsy, Breast cancer, Meta-analysis, Systematic review, Breast Neoplasms, Lymphatic metastasis

\section{Background}

Internal mammary lymph node (IMN) is an important part of lymphatic drainage of breast cancer. In the last few decades, a lot of effort has been made to optimize the management of IMN. It was well established in the 1970s that extended radical mastectomy (ERM) including routine dissection of IMN would not improve disease control whiles bring about serious complications [1]. Multiple studies have shown that patients with IMN metastasis had a worse outcome [2-4]. The metastasis of IMN has been incorporated into the American Joint Committee on Cancer (AJCC) cancer staging system of

\footnotetext{
* Correspondence: lincongyao@znhospital.cn

'Department of Thyroid and Breast Surgery, Zhongnan Hospital of Wuhan University, 169 DongHu Road, Wuhan 430071, People's Republic of China Full list of author information is available at the end of the article
}

breast cancer since the 6th edition as an evidence of increased tumor burden.

The MA.20 [5] and the EORTC 22922-10925 trial [6] and a French trial [7] have found out that radiation therapy (RT) to IMN basin will improve patients' survival. The National Comprehensive Cancer Network (NCCN) Breast Cancer Clinical Practice Guideline has raised the level of evidence for the recommendation of RT when there is a high risk of IMN involvement. However, it was not reliable to identify patients with IMN metastasis by clinical factors such as tumor location or axillary lymph nodes (AXN) status. As a result, patients with actual IMN involvement might escape RT, while patients without IMN metastasis would undergo unnecessary RT.

Recent studies have demonstrated that IMN status can be safely determined through sentinel lymph node 
biopsy (SLNB) technique $[8,9]$. But questions remain whether IMN-SLNB should be performed routinely and which patient would benefit the most. To date, there have been a growing number of studies trying to answer these questions. However, many of these researches have been retrospective in nature and not much large randomized trials were performed. A previous systematic review in 2012 included 18 studies reporting that positive IMN are reliable predictors of axillary lymph node involvement. Nonetheless, this study suffered from a paucity of data and small case series and case reports have to be included [10]. In 2013, another meta-analysis enrolled 15 articles did not specify SLNB as an inclusion criterion while evaluating IMN metastasis-associated outcomes [11]. Since the publication of those two metaanalyses, more studies have become available, which justifies a new systematic review.

Our objective is to conduct a quantitative analysis of current evidence and provide important insights into the IMN management.

\section{Material and methods Study identification}

We performed a computer-based literature search of PubMed/MEDLINE, Clinical trials, EMBASE, Scopus, Cochrane electronic databases, and Google Scholar. The core search strategy was as follows: internal mammary [All Fields] AND ("lymph nodes" [MeSH Terms] OR "lymph" [All Fields] AND "nodes" [All Fields]) OR "lymph nodes" [All Fields]). The following search terms were also used: extra-axillary lymph nodes, intramammary lymph node, intraMSLN, and retromammary lymph nodes. The search was neither limited by language nor by date. The full texts of all the relevant studies were retrieved. A manual search was also conducted to scan the reference list of relevant articles in order to identify additional articles. The last search was made in April 2019.

\section{Eligibility criteria}

Studies were included in our systematic review if they fulfilled the following criteria: published research articles including patients underwent IMN-SLNB and reported the status of IMN as well as axillary lymph nodes (AXN). We excluded case reports, reviews, comments, letter to editors, correspondence, conference posters. We also excluded studies that included patients receiving neoadjuvant therapy, and studies reported extra-axillary lymph nodes other than IMN, and studies that did not report enough adequate data or pathological results.

\section{Data extractions and management}

Data was extracted independently by two authors on a predefined form. In addition to IMN and AXN status, information on potentially confounding study-level characteristics (stage, age, length of follow-up, SLNB technique, tumor histology, tumor size, and tumor location) was also extracted with the intention of performing possible subgroup analyses. Differences between reviewers were resolved by discussion. When certain events were not explicitly stated in the study, information was calculated through available data. Several authors were contacted for unpublished data through emails when there was not enough data amenable.

\section{Data synthesis and meta-analysis}

We calculated the pooled proportion of positivity of IMN-SLNB with the corresponding 95\% confidence intervals (CI). The statistical heterogeneity across studies was tested by $Q$ statistics. An alpha value of 0.5 was taken to indicate between-study heterogeneity, which is represented by $I^{2}$ values. Significant between-group heterogeneity was observed and random-effects models were applied. Pooled estimated proportion of IMNSLNB was presented by forest plots. We also calculate the odds ratio (OR) of 4 subgroups (IMN+/AXN+, IMN+/AXN-, IMN-/AXN-, IMN-/AXN-). The OR was further combined in order to explore the association of IMN and AXN. Statistical analyses were conducted in STATA 14 software (StataCorp LP, College Station, TX).

\section{Results}

Literature search and study identification

A systematic literature search was performed to identify studies that met the predefined eligibility criteria. The search yielded 215 articles. Full texts of 99 articles were retrieved. We defined studies reporting no more than 4 cases as small case series. Small case series and reviews, correspondence and conference posts were excluded. Studies based on the same population or on human cadavers were excluded. Furthermore, studies that enrolled neoadjuvant treatment or did not report enough data were excluded. Finally, 18 studies (with 2427 patients) were included in this systematic review. The process of research screening and selection is shown in a flow chart (Fig. 1).

\section{Study characteristics}

Table 1 showed an overview of the characteristics of all selected articles. The majority of them was retrospective cohort studies and employed more than one method of SLNB. It should be noted that not all included studies reported the visualization rate and success rate of IMNSLNB. Interestingly, both the visualization and success rate seemed to vary widely from study to study. IMN was visualized in 20\% of patients (range 5.4-87.9\%). IMN-SLNB was successful in $90.3 \%$ patients (range $45-$ $97.3 \%)$. In spite of this, the positivity rate of IMN-SLNB 


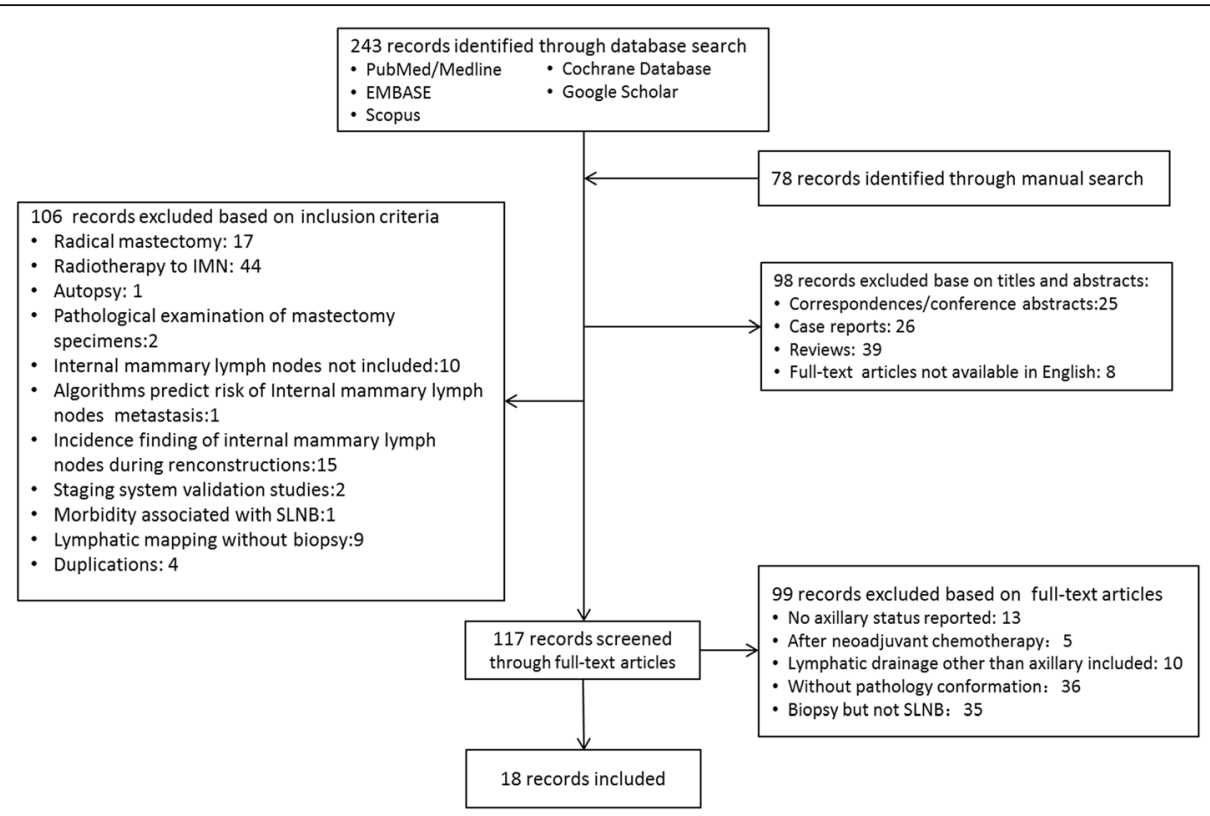

Fig. 1 Flowchart of included studies

was rather stable (range 8.8-25\%). The highest metastatic rate was reported by Avisar et al. with $44.1 \%$ of the lesions located in the central area [12]. The lowest positive rate was reported by an early innovative study by Galimberti et al. when IMN were sampled without the help of a gamma probe [16].

Meta-analysis The pooled estimated of IMN-SLNB positivity rate was $15 \%$ (95\% CI 12-17\%) (Fig. 2). Significant between-group heterogeneity was observed and random-effects models were applied. Subgroup analysis was also performed. Included studies were further stratified by tumor stage (with or without metastasis) and publication year. Surprisingly, the pooled positive rate of IMN-SLNB did not change significantly according to our subgroup analysis (Figs. 3 and 4).

We also explored the correlations of IMN and AXN metastasis. As it is shown in Fig. 5, our results indicated that axillary lymph node metastasis is a strong predictor of IMN involvement (OR 6.01, 95\% CI 3.49, 10.34). The metastasis of IMN predicts the metastasis of AXN, and vice versa.

\section{Bias assessment}

Funnel plot was employed to assess publication bias. As is shown in Fig. 6, there was no significant publication bias.

\section{Discussions}

Whether we should biopsy internal mammary nodes or not has been a matter of debate for a few decades. The argument was brought up during the era of ERM when worse prognosis was noticed in patients with IMN involvement. However, the IMN dissection as part of ERM was later abandoned due to lack of survival benefit. The introduction of SLNB technique has dramatically changed the clinical management of AXN. It was also applied to evaluate IMN status since it was a relatively safe procedure compared to ERM. At first, IMN-SLNB technique was not quite reliable. Many earlier studies reported a very low incidence let alone metastasis rate of IMN. Moreover, IMN-SLNB carried a certain risk of complications. So the omission of IMN-SLNB was suggested by many researchers. Qiu et al. modified the IMN-SLNB technique and reported a visualization rate of $75 \%$ with a success rate of $98 \%$ and a serious complication rate of 9.1\% [28].

The incidence of IMN metastasis might be underestimated. The incidence of IMN detected by modern imaging technique such as MRI or CT is around $45 \%$, but it was hard to rule out reactive hyperplasia and the detection criteria varied greatly $[29,30] . P E T / C T$ is very promising in differentiating IMN SLNs from mediastinal sentinel lymph nodes (SLNs). The latter is a frequent site of tumor migration and cancer relapse, but it is unable to be biopsied without thoracic surgery [31].

Recently, several large prospective studies have demonstrated the survival benefit of RT to IMN basin [5-7]. Based on these solid evidence, the 2016 NCCN guideline has changed the level of recommendation of IMN-RT to category $2 \mathrm{~A}$ when there is a high risk of IMN involvement. However, there are other studies that failed to show a survival benefit with IMN-RT [32,33]. This controversy might be explained by three reasons: firstly, a pathological confirmation of IMN status was not always 
Table 1 Characteristic of eligible studies in the meta-analysis

\begin{tabular}{|c|c|c|c|c|c|c|c|c|c|c|c|}
\hline \multirow[t]{2}{*}{ Author } & \multirow[t]{2}{*}{ Year } & \multirow[t]{2}{*}{ Country } & \multirow{2}{*}{$\begin{array}{l}\text { No. of } \\
\text { patients }\end{array}$} & \multirow[t]{2}{*}{ Nature } & \multirow[t]{2}{*}{ Stage } & \multirow{2}{*}{$\begin{array}{l}\text { Lymphatic mapping } \\
\text { technique }\end{array}$} & \multirow{2}{*}{$\begin{array}{l}\text { Positivity } \\
\text { rate (\%) }\end{array}$} & \multicolumn{2}{|l|}{$\mathrm{IMN+}$} & \multicolumn{2}{|l|}{ IMN- } \\
\hline & & & & & & & & $\begin{array}{l}\mathrm{AXN+} \\
n(\%)\end{array}$ & $\begin{array}{l}\text { AXN- } \\
n(\%)\end{array}$ & $\begin{array}{l}\mathrm{AXN+} \\
n(\%)\end{array}$ & $\begin{array}{l}\text { AXN- } \\
n(\%)\end{array}$ \\
\hline Avisar [12] & 2008 & American & 31 & Prospective & T1-3 No & $\begin{array}{l}\text { Gamma probe or blue } \\
\text { dye or } \mathrm{LS}\end{array}$ & 25 & $5(17.86)$ & $2(7.14)$ & $5(17.86)$ & $16(27.59)$ \\
\hline Bourre [13] & 2009 & France & 161 & Petrospective & T1 NO & $\begin{array}{l}\text { Gamma probe and blue } \\
\text { dye and LS }\end{array}$ & 11 & $11(6.83)$ & $7(4.34)$ & $7(4.34)$ & $135(86.25)$ \\
\hline Carcoforo [14] & 2006 & Italy & 65 & Retrospective & NO & $\begin{array}{l}\text { Gamma probe and blue } \\
\text { dye and LS }\end{array}$ & 15.40 & $3(10.34)$ & $1(3.45)$ & $4(13.79)$ & $21(72.41)$ \\
\hline Caudle [8] & 2014 & American & 71 & Retrospective & NO & $\begin{array}{l}\text { Gamma probe and blue } \\
\text { dye and LS }\end{array}$ & 15 & $4(5.63)$ & $7(9.86)$ & $12(16.90)$ & $48(67.61)$ \\
\hline Cong [15] & 2016 & China & 145 & Prospective & I-IV & $\begin{array}{l}\text { Gamma probe and LS } \\
\text { and fluorescence }\end{array}$ & 12.40 & $11(7.01)$ & $8(5.10)$ & $59(37.58)$ & $79(50.32)$ \\
\hline Galimberti [16] & 2002 & Italy & 160 & Retrospective & I-IV & LS & 8.80 & $10(6.25)$ & $4(2.50)$ & $45(28.13)$ & $101(63.13)$ \\
\hline Generich [9] & 2014 & American & 122 & Retrospective & Tis-T2 No & Gamma probe and LS & 10 & $10(9.26)$ & $2(1.85)$ & $20(18.52)$ & $76(70.37)$ \\
\hline $\mathrm{He}[17]$ & 2010 & China & 94 & Retrospective & T1-2 No & $\begin{array}{l}\text { Gamma probe and LS } \\
\text { and carbon nanoparticles }\end{array}$ & 24 & $19(20.21)$ & $4(4.26)$ & $10(10.64)$ & $61(64.89)$ \\
\hline Heuts [18] & 2009 & Netherlands & 139 & Retrospective & $\mathrm{cNO}$ & $\begin{array}{l}\text { Gamma probe and blue } \\
\text { dye and LS }\end{array}$ & 22 & $22(15.83)$ & $9(6.47)$ & $43(30.94)$ & $65(46.76)$ \\
\hline Lee [19] & 2013 & Korea & 31 & Retrospective & cNO & NR & 20.00 & $1(10.00)$ & $1(10.00)$ & $0(0.00)$ & $8(80.00)$ \\
\hline Leidenius [20] & 2006 & Finland & 122 & Retrospective & T1-2 No & Gamma probe and LS & 15 & $10(7.25)$ & $8(5.80)$ & $28(20.29)$ & $92(66.67)$ \\
\hline Mansel [21] & 2004 & UK & 31 & $\mathrm{RCT}$ & cNO & Gamma probe and LS & 13 & $2(6.45)$ & $2(6.45)$ & $15(48.39)$ & $12(38.71)$ \\
\hline Maraz [22] & 2014 & Hungary & 77 & Retrospective & T1-3 No & Blue dye and LS & 18 & $2(2.60)$ & $12(15.58)$ & $14(18.18)$ & $49(63.64)$ \\
\hline Ozmen [23] & 2015 & Turkey & 72 & Retrospective & $\mathrm{cNO}$ & LS & 14 & $9(12.50)$ & $1(1.39)$ & $24(33.33)$ & $38(52.78)$ \\
\hline Piato [24] & 2016 & Brazil & 20 & Prospective & $|-| \mid$ & LS and PET-CT & 21 & $2(10.53)$ & $2(10.53)$ & $1(5.26)$ & $14(73.68)$ \\
\hline Postma [25] & 2012 & Netherlands & 86 & Retrospective & T1-2 No & $\begin{array}{l}\text { Gamma probe and blue } \\
\text { dye and LS }\end{array}$ & 16.00 & $7(8.14)$ & $7(8.14)$ & $25(29.07)$ & $47(54.65)$ \\
\hline Qi [26] & 2018 & China & 337 & Retrospective & Mo & NR & 18.70 & $62(18.40)$ & $1(0.30)$ & $132(39.17)$ & $142(42.14)$ \\
\hline Veronesi [27] & 2008 & Italy & 663 & Retrospective & I-IV & Gamma probe and LS & 10.30 & $51(7.69)$ & $17(2.56)$ & $182(27.45)$ & $413(62.29)$ \\
\hline
\end{tabular}

$A X N$ axillary lymph nodes, IMN internal mammary lymph node, $L S$ lymphoscintigraphy, $M O$ no presence of distant metastasis, NO no presence of lymphatic involvement, NR not reported, PET-CT positron emission tomography-computed tomography, $R C T$ randomized clinical trial, Tis carcinoma in situ, $n$ number of patients, + positive, presence of lymph node metastasis, - negative, no presence of lymph node metastasis

available in these studies. Risk stratification of IMN metastasis by clinical factors like tumor location is not always reliable. A recent study validated the hypothesis that IMN SLNs receive lymphatic drainage from all four quadrants of breast, which was consistent with results of previous studies of ERM [34]. Secondly, it was difficult to single out the survival benefit of RT to IMN basin apart from routine RT. Thirdly and most importantly, patients with IMN metastasis are still a heterogeneous sub-group of breast cancer. The 8th edition of AJCC staging system further stratified IMN-positive patients based on other prognostic factors such as hormone receptor and human epidermal growth factor receptor-2 (HER2) status, which might lead to a more accurate prognosis prediction [35]. So, it would be of great value if the status of IMN in high-risk patients could be assessed pre-operatively and adjuvant treatment plan especially radiotherapy could be tailed.

Our results showed the pooled estimated rate of metastasis IMN was $15 \%$ which was in consistence with recent reports [36]. Studies have found out that there are several factors that can affect the positivity rate of IMN-
SLNB, such as tumor location, tumor stage, age, and systematic therapy. The mapping and detection procedure will also affect the visualization of IMN. The majority of our included studies employed more than more one mapping techniques. Despite the variation of visualization rate, publication year and the presence of distant metastasis, the positivity rate of IMN-SLNB was rather consistent. Our study also showed that there was a significant correlation between IMN and AXN. Patients with AXN involvement are 6 times more likely to have IMN involvement. This correlation of AXN and IMN has been noticed since the era of ERM [37]. Our results add to the growing body of evidence for the use of IM-SLNB in patients with axillary involvements [26]. However, these results should be interpreted with caution. Future work is required to verify if IMN-SLNB should be recommended in case of AXN involvement and be omitted when AXN is negative.

Our study might be subjected to several limitations. We are trying to draw a contemporary picture of IMN status in the era of SLNB. Yet, our characteristic analysis was based on data extracted from a majority of retrospective studies and therefore selective bias was inevitable. On the 


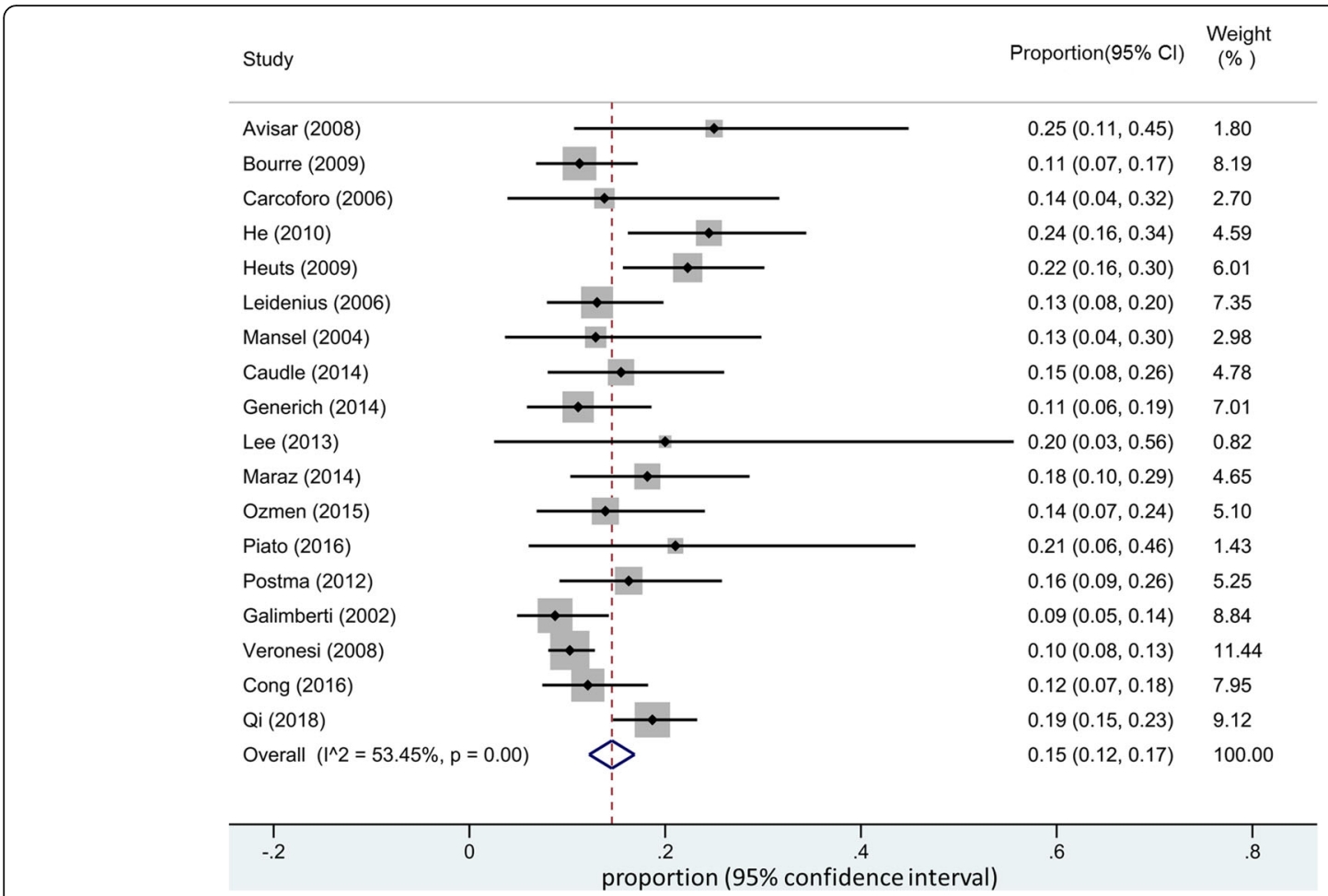

Fig. 2 Pooled estimate of IMN-SLNB positivity rate

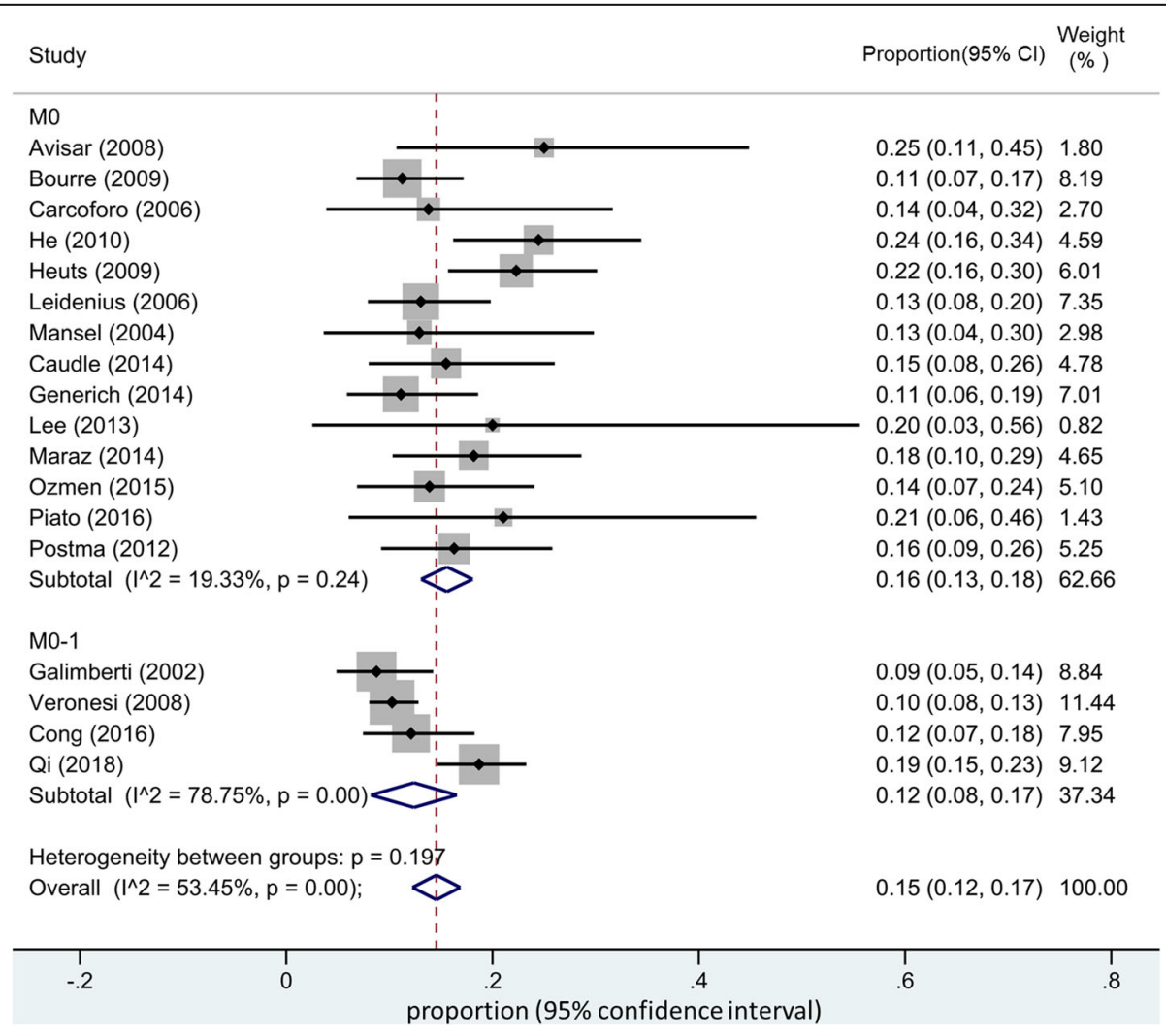

Fig. 3 Subgroup analysis of the pooled estimate of IMN-SLNB positivity rate in patients with or without distant metastasis 


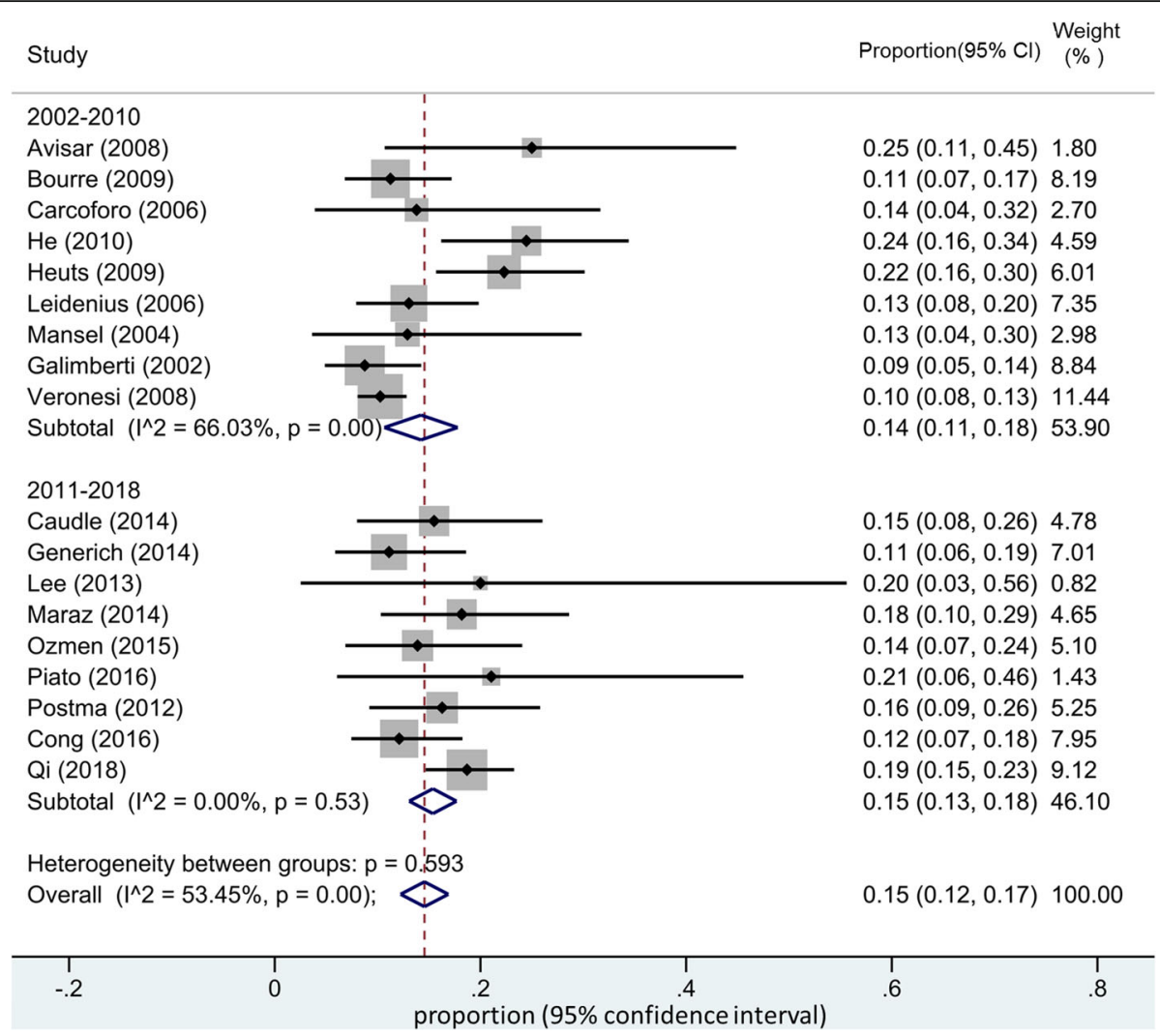

Fig. 4 Subgroup analysis of the pooled estimate of IMN-SLNB positivity rate stratified by publication year

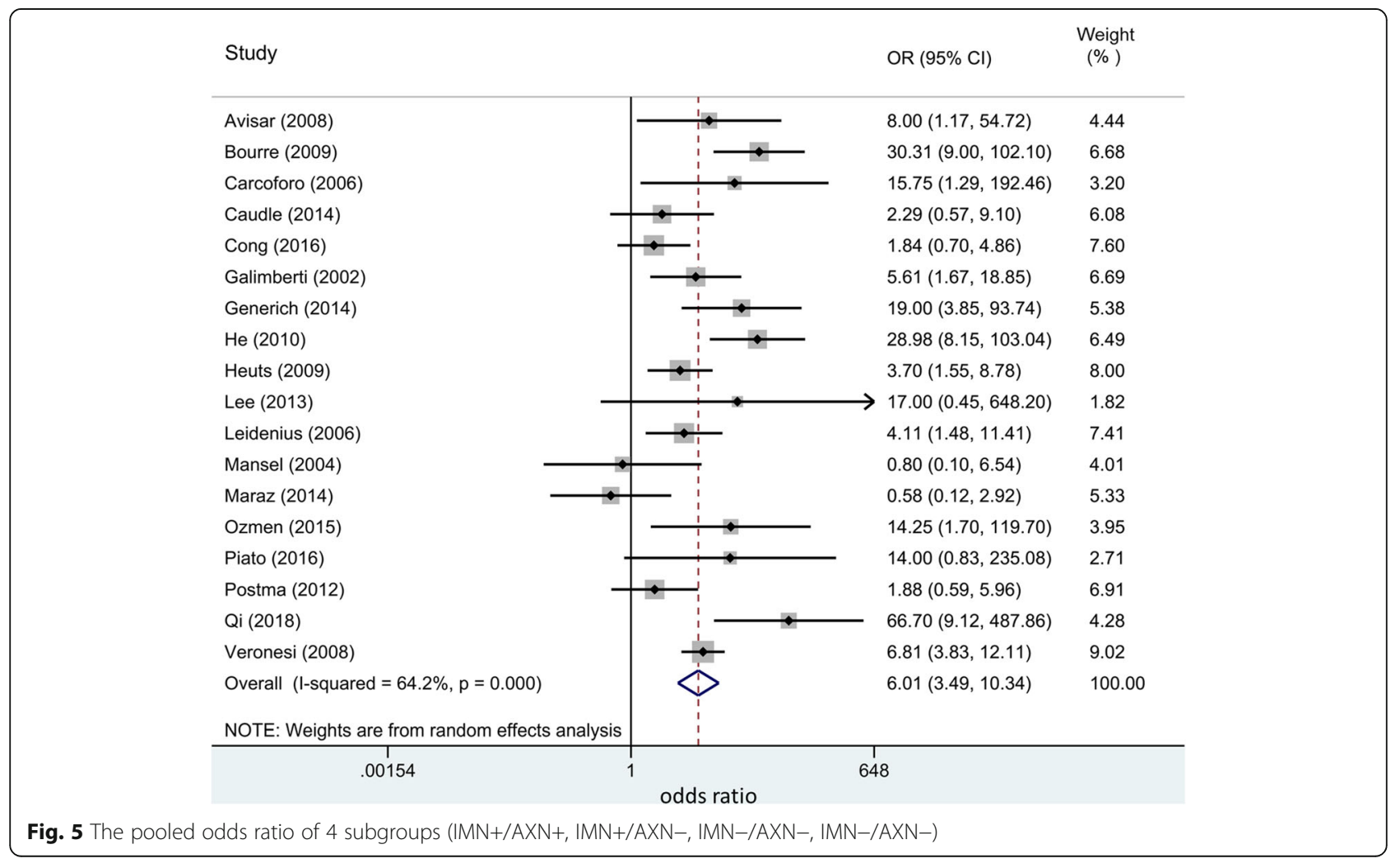




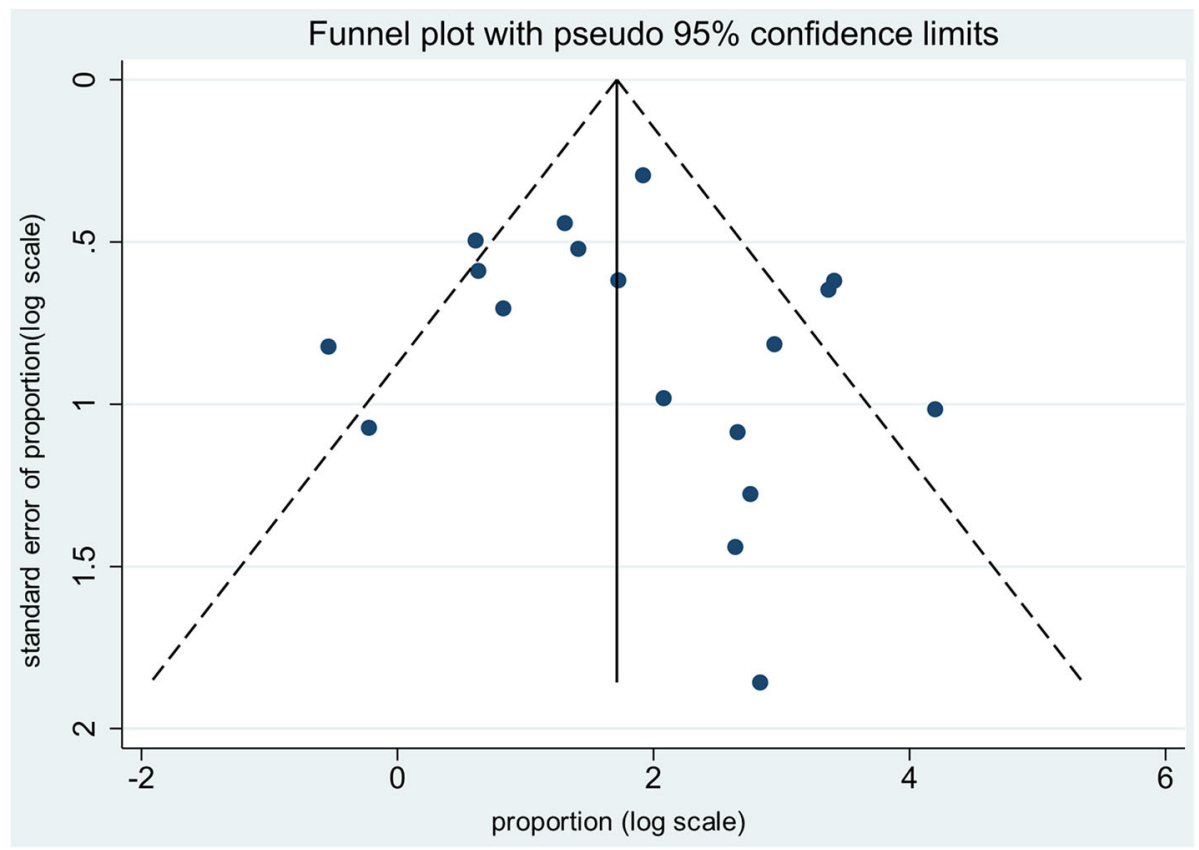

Fig. 6 Funnel plot to assess publication bias

other hand, we conducted a rigorous screening. We were able to exclude case reports and very small case serious. However, there are some other means to investigate IMN that was not included in our study. For instance, IMN biopsy during breast reconstructions is not very uncommon, and IMN status can also be determined by fine-needle aspiration or core biopsy or thoracic surgery. Finally, only 3 of our included studies reported survivals with insufficient data; therefore, a pooled survival analysis cannot be performed and the impact of positive IM-SLNB on survival cannot be determined.

\section{Conclusions}

Our results revealed the estimated IMN-SLNB positivity rate. The metastasis of axillary lymph nodes was a major predictive factor of IMN involvement. Patients with positive axillary lymph nodes have a higher risk of internal lymph nodes metastasis and IM-SLNB might be considered. More prospective randomized clinical trials should be conducted to fully address this issue.

\section{Abbreviations}

-: Negative, no presence of lymph node metastasis; +: Positive, presence of lymph node metastasis; AJCC: American Joint Committee on Cancer; AXN: Axillary lymph node; Cl: Confidence interval; ERM: Extended radical mastectomy; IMN: Internal mammary lymph node; LS: Lymphoscintigraphy; MO: No presence of distant metastasis; $n$ : Number of patients; No: No presence of lymphatic involvement; NCCN: National Comprehensive Cancer Network; NR: Not reported; OR: Odds ratio; PET-CT: Positron emission tomography-computed tomography; RCT: Randomized clinical trial; RT: Radiation therapy; SLNB: Sentinel lymph node biopsy; Tis: Carcinoma in situ

\section{Authors' contributions}

All authors participated in the study. JG and CYL performed the literature search and the extraction of data. JG and YFY performed the data analysis. CYL and XT participated in the interpretation of data and the writing of the manuscript. GSW revised the article for important intellectual content. All authors read and approved the final manuscript.

\section{Funding}

This work was supported by Wuhan University. The funding projects are Independent Research Projects of Wuhan University (grant no. 2042019kf0157) and Start-up Funds for Scientific Research of High-level Talents ( grant no. 120162301192633).

Availability of data and materials

All data generated or analyzed during this study are included in this published article.

Ethics approval and consent to participate Not applicable.

\section{Consent for publication}

Not applicable.

\section{Competing interests}

The authors declare that they have no competing interests.

\section{Author details}

'Department of Thyroid and Breast Surgery, Zhongnan Hospital of Wuhan University, 169 DongHu Road, Wuhan 430071, People's Republic of China. ${ }^{2}$ Department of Clinical Epidemiology, Aarhus University, Olof Palmes Allé 43-45, 8200 Aarhus N, Denmark. ${ }^{3}$ School of Mechanical Engineering and Electronic Information, China University of Geosciences, 388 Lumo Road, Wuhan 430074, People's Republic of China. 
Received: 10 June 2019 Accepted: 29 July 2019 Published online: 05 August 2019

\section{References}

1. Veronesi U, Marubini E, Fau - Mariani L, Mariani L, Fau - Valagussa P, Valagussa P, Fau - Zucali R, Zucali R. The dissection of internal mammary nodes does not improve the survival of breast cancer patients. 30-year results of a randomised trial. Eur J Cancer. 1999;35(9):1320-5.

2. Shen J, Hunt KK, Mirza NQ, Krishnamurthy S, Singletary SE, Kuerer HM, et al. Intramammary lymph node metastases are an independent predictor of poor outcome in patients with breast carcinoma. Cancer. 2004;101(6):1330-7.

3. Hogan BV, Peter MB, Shenoy H, Horgan K, Shaaban A. Intramammary lymph node metastasis predicts poorer survival in breast cancer patients. Surg Oncol. 2010;19(1):11-6

4. Sugg SL, Ferguson DJ, Posner MC, Heimann R. Should internal mammary nodes be sampled in the sentinel lymph node era? Ann Surg Oncol. 2000; 7(3):188-92.

5. Whelan TJ, Olivotto IA, Parulekar WR, Ackerman I, Chua BH, Nabid A, et al. Regional nodal irradiation in early-stage breast cancer. New England J Med. 2015;373(4):307-16.

6. Poortmans PM, Collette S, Kirkove C, Van Limbergen E, Budach V, Struikmans $H$, et al. Internal mammary and medial supraclavicular irradiation in breast cancer. New England J Med. 2015;373(4):317-27.

7. Hennequin C, Bossard N, Servagi-Vernat S, Maingon P, Dubois JB, Datchary J, et al. Ten-year survival results of a randomized trial of irradiation of internal mammary nodes after mastectomy. Int J Rad Oncol Biol Physic. 2013;86(5):860-6.

8. Caudle AS, Yi M, Hoffman KE, Mittendorf EA, Babiera GV, Hwang RF, et al. Impact of identification of internal mammary sentinel lymph node metastasis in breast cancer patients. Ann Surg Oncol. 2014;21(1):60-5.

9. Gnerlich JL, Barreto-Andrade JC, Czechura T, John JR, Turk MA, Kennedy TJ, et al. Accurate staging with internal mammary chain sentinel node biopsy for breast cancer. Ann Surg Oncol. 2014;21(2):368-74

10. Abdullgaffar B, Gopal P, Abdulrahim M, Ghazi E, Mohamed E. The significance of intramammary lymph nodes in breast cancer: a systematic review and meta-analysis. Int J Surg Pathol. 2012:20(6):555-63.

11. Li Z, Gu X, Tong J, Liu B, Sun L, Gao X, et al. A meta-analysis of internal mammary lymph node metastasis in breast cancer patients. Onkologie. 2013;36(12):747-52.

12. Avisar E, Molina MA, Scarlata M, Moffat FL. Internal mammary sentinel node biopsy for breast cancer. Am J Surg. 2008;196(4):490-4.

13. Bourre JC, Payan R, Collomb D, Gallazzini-Crepin C, Calizzano A, Desruet MD, et al. Can the sentinel lymph node technique affect decisions to offer internal mammary chain irradiation? Eur J Nucl Med Mol Imaging. 2009; 36(5):758-64.

14. Carcoforo P, Sortini D, Feggi L, Feo CV, Soliani G, Soliani G, Panareo S, Corcione $S$, et al. Clinical and therapeutic importance of sentinel node biopsy of the internal mammary chain in patients with breast cancer: a single-center study with long-term follow-up. Ann Surg Oncol. 2006; 10689265 (Print)

15. Cong BB, Qiu PF, Liu YB, Zhao T, Chen P, Cao XS, et al. Validation study for the hypothesis of internal mammary sentinel lymph node lymphatic drainage in breast cancer. Oncotarget. 2016;7(27):41996-2006.

16. Galimberti V, Veronesi P, Arnone P, De Cicco C, Renne G, Intra M, et al. Stage migration after biopsy of internal mammary chain lymph nodes in breast cancer patients. Ann Surg Oncol. 2002;9(9):924-8.

17. He Q, Zhuang D, Tian J, Zheng L, Fan Z, Li X. Surgical approach to internal mammary sentinel node biopsy. J Invest Surg. 2010;23(6):321-6.

18. Heuts EM, van der Ent FW, von Meyenfeldt MF, Voogd AC. Internal mammary lymph drainage and sentinel node biopsy in breast cancer - A study on 1008 patients. Eur J Surg Oncol. 2009;35(3):252-7.

19. Lee SK, Kim S, Choi MY, Kim J, Lee J, Jung SP, et al. The clinical meaning of intramammary lymph nodes. Oncology. 2013;84(1):1-5.

20. Leidenius MH, Krogerus LA, Toivonen TS, Leppanen EA, Von Smitten K.A.J. The clinical value of parasternal sentinel node biopsy in breast cancer. 2006. (1068-9265 (Print))

21. Mansel RE, Goyal A, Newcombe RG. Internal mammary node drainage and its role in sentinel lymph node biopsy: the initial ALMANAC experience. Clin Breast Cancer. 2004;5(4):279-84 discussion 85-6.
22. Maráz R, Boross G, Pap-Szekeres J, Rajtár M, Ambrózay E, Cserni G. Internal mammary sentinel node biopsy in breast cancer. is it indicated? Pathol Oncol Res. 2014;20(1):169-77.

23. Ozmen V, Ozcinar B, Bozdogan A, Eralp Y, Yavuz E, Dincer M. The effect of internal mammary lymph node biopsy on the therapeutic decision and survival of patients with breast cancer. Eur J Surg Oncol. 2015:41(10):1368-72.

24. Piato JR, Filassi JR, Dela Vega AJ, Coura-Filho GB, Aguiar FN, Porciuncula LM, et al. SPECT-CT-Guided thoracoscopic biopsy of sentinel lymph nodes in the internal mammary chain in patients with breast cancer: a pilot study. Innovations (Phila). 2016;11(2):94-8

25. Postma EL, van Wieringen S, Hobbelink MG, Verkooijen HM, van den Bongard HJ, Borel Rinkes $\mathrm{H}$, et al. Sentinel lymph node biopsy of the internal mammary chain in breast cancer. Breast Cancer Res Treat. 2012; 134(2):735-41.

26. Qi X-w, J-z D, Tang P, Liu X, He Q-q, Zhong L, et al. Clinical significance of internal mammary lymph node metastasis for breast cancer: Analysis of 337 breast cancer patients. Surg Oncol. 2018;27(2):185-91.

27. Veronesi U, Arnone P, Veronesi P, Galimberti V, Luini A, Rotmensz N, Botteri $E_{\text {, et }}$ al. The value of radiotherapy on metastatic internal mammary nodes in breast cancer. Results on a large series. 2008. (1569-8041 (Electronic)).

28. Qiu PF, Cong BB, Zhao RR, Yang GR, Liu YB, Chen P, et al. Internal mammary sentinel lymph node biopsy with modified injection technique: high visualization rate and accurate staging. Medicine. 2015;94(41):e1790

29. Ray KM, Munir R, Wisner DJ, Azziz A, Holland BC, Kornak J, et al. Internal mammary lymph nodes as incidental findings at screening breast MRI. Clin Imaging. 2015;39(5):791-3.

30. Savaridas SL, Spratt JD, Cox J. Incidence and potential significance of internal mammary lymphadenopathy on computed tomography in patients with a diagnosis of primary breast cancer. Breast Cancer. 2015;9:59-65.

31. Serrano-Vicente J, Rayo-Madrid Jl, Dominguez-Grande ML, Infante-Torre JR, Garcia-Bernardo L, Moreno-Caballero M, et al. Role of SPECT-CT in breast cancer sentinel node biopsy when internal mammary chain drainage is observed. Clin Transl Oncol. 2016:18(4):418-25.

32. Fowble B, Hanlon A, Freedman G, Nicolaou N, Hoffman J, Sigurdson E, et al. Internal mammary node irradiation neither decreases distant metastases nor improves survival in stage I and I| breast cancer. Int J Radiat Oncol Biol Phys. 2000:47(4):883-94

33. Olson RA, Woods R, Speers C, Lau J, Lo A, Truong PT, Tyldesley S, et al. Does the intent to irradiate the internal mammary nodes impact survival in women with breast cancer? A population-based analysis in British Columbia. (1879-355X (Electronic)).

34. Borgstein PJ, Meijer S, Pijpers RJ, van Diest PJ. Functional lymphatic anatomy for sentinel node biopsy in breast cancer: echoes from the past and the periareolar blue method. (0003-4932 (Print)).

35. Joo JH, Kim SS, Son BH, S DOA, Jung JH, Choi EK, et al. Evaluation of the prognostic stage in the 8th edition of the American Joint Committee on Cancer in Patients with Breast Cancer and Internal Mammary Lymph Node Metastasis: Electronic; 2018. p. 1791-7530.

36. Hindie E, Groheux D, Hennequin C, Zanotti-Fregonara P, Vercellino L, Berenger $\mathrm{N}$, et al. Lymphoscintigraphy can select breast cancer patients for internal mammary chain radiotherapy. Int J Radiat Oncol Biol Phys. 2012; 83(4):1081-8.

37. Huang O, Wang L, Fau - Shen K, Shen K, Fau - Lin H, Lin H, Fau - Hu Z, Hu Z, Fau - Liu G, Liu G, Fau - Wu J, et al. Breast cancer subpopulation with high risk of internal mammary lymph nodes metastasis: analysis of 2,269 Chinese breast cancer patients treated with extended radical mastectomy. p. 0167-6806.

\section{Publisher's Note}

Springer Nature remains neutral with regard to jurisdictional claims in published maps and institutional affiliations. 